\title{
Convex Disks Can Cover Their Shadow
}

\author{
Géza Kós and Jenó Törôcsik \\ Department of Mathematics, Eötrös University, \\ Múzeum krt. 6-8, H-1088 Budapest, Hungary
}

\begin{abstract}
We give a short proof of the fact that given any two-dimensional convex disk in three-space and an orthogonal projection $C^{\prime}$ of $C$ on a plane then there is a congruent copy of $C$ which contains $C^{\prime}$.
\end{abstract}

We give a short proof for the next theorem conjectured independently by Zalgaller [1] and Pach [2] and proved independently by Kowaljov [3] and Debrunner and Mani-Levitska [4].

Theorem. Any orthogonal projection $C^{\prime}$ of a two-dimensional convex compact set $C$ in $R^{3}$ onto a two-dimensional subspace $S$ can be covered by some congruent copy of $C$.

Proof. It is clear that there is a congruent copy $C_{1}$ of $C$ in the plane $S$ and there is an orthogonal basis $\{x, y\}$ in $S$ such that $C^{\prime}$ is the image of $C_{1}$ under a linear map which maps $x$ to $x$ and $y$ to $\lambda y$ with some $\lambda \in[0,1]$. We denote the image of point $P$ under this map by $P^{\prime}$.

It would suffice to find parallel lines $e$ and $f$, tangent to $C_{1}$ at points $E$ and $F$ such that, for each point $P$ of $C_{1}, \angle P E F \geq \angle P^{\prime} E^{\prime} F^{\prime}$ and $\angle P F E \geq \angle P^{\prime} F^{\prime} E^{\prime}$. Indeed, in this case, any orientation preserving isometry $i$ of $R^{2}$ which maps the segment $E^{\prime} F^{\prime}$ onto the segment $E F$ (with $E^{\prime}$ contained in the segment $E F^{\prime}$ ) will map $C^{\prime}$ onto $C_{1}$. In fact, for each $P \in C_{1}, i\left(P^{\prime}\right) \in i\left(\triangle P^{\prime} E^{\prime} F^{\prime}\right) \subset \triangle P E F \subset C_{1}$ will hold (Fig. 1).

In order to find $e$ and $f$ let $\vec{x}$ be the direction determined by $x$. Let $\angle \vec{d}_{1} \vec{d}_{2}$ be the angle between directions $\vec{d}_{1}$ and $\vec{d}_{2}$ measured by rotating $\vec{d}_{1}$ counterclockwise into $\vec{d}_{2}$. Let $\alpha=\arctan (1 / \sqrt{\lambda})$. For every direction $\vec{d}$ with $|\angle \vec{x} \vec{d}| \leq \alpha$ define the direction $\vec{d}^{*}$ with $\tan (\angle \vec{x} \vec{d}) \tan \left(\angle \vec{x} \vec{d}^{*}\right)=1 / \lambda$ (Fig. 2). Easy computation shows that $\angle \vec{d} \vec{d}^{*}=\angle \vec{d}^{\prime} \vec{d}^{*}$. Moreover, if $|\angle \vec{x} \vec{d}| \leq \alpha$ and $\vec{d}_{0}$ is between $\vec{d}$ and $\vec{d}^{*}$ or between $\vec{d}$ and $\vec{d}^{*}$ (where $\vec{d}^{*}$ is the direction opposite to $\vec{d}^{*}$ ), then $\angle \vec{d} \vec{d}_{0} \geq \angle \vec{d}^{\prime} \vec{d}_{0}^{t}$. 


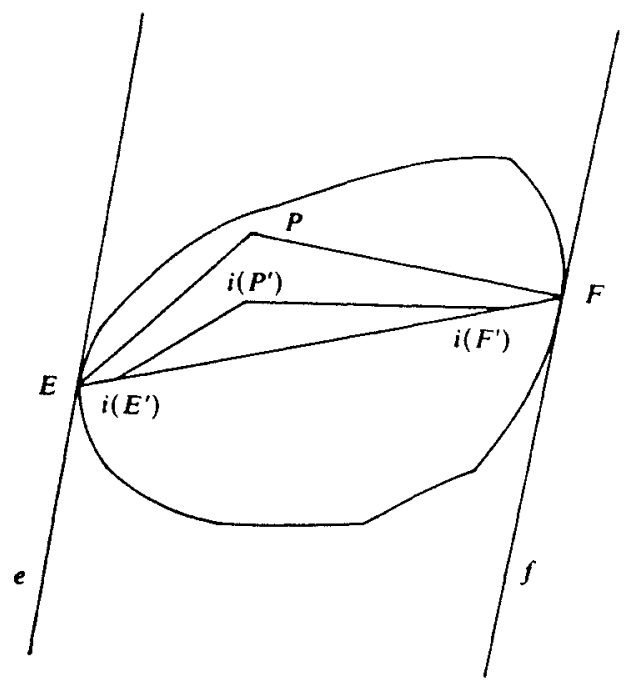

Fig. 1

So it suffices to find parallel lines $e$ and $f$, tangent to $C_{1}$ such that $|\angle \vec{x} \overrightarrow{E F}| \leq \alpha$ and $\overrightarrow{E F}^{*}=\vec{e}=\vec{f}$ holds.

Define a decreasing continuous function $g$ from $[-\alpha, \alpha]$ to $[0, \pi]$ such that $g(-\alpha)=\pi, g(\alpha)=0$, and $g(\angle \vec{x} \vec{d})=\angle \vec{d} \vec{d} *$ for every $\vec{d}$ with $|\angle \vec{x} \vec{d}| \leq \alpha$. Let $G$ be the graph of $g$ (Fig. 3). Define $H \subset[-\alpha, \alpha] \times[0, \pi]$ such that $(\mu, \nu) \in H$ iff there

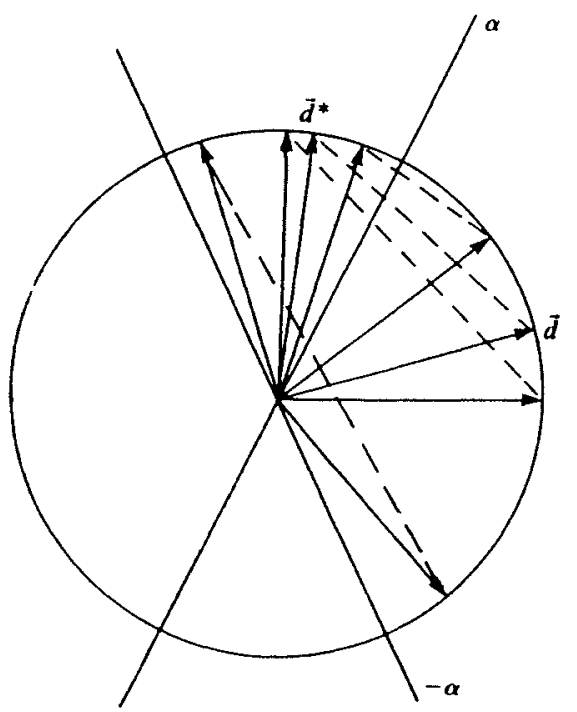

Fig. 2 


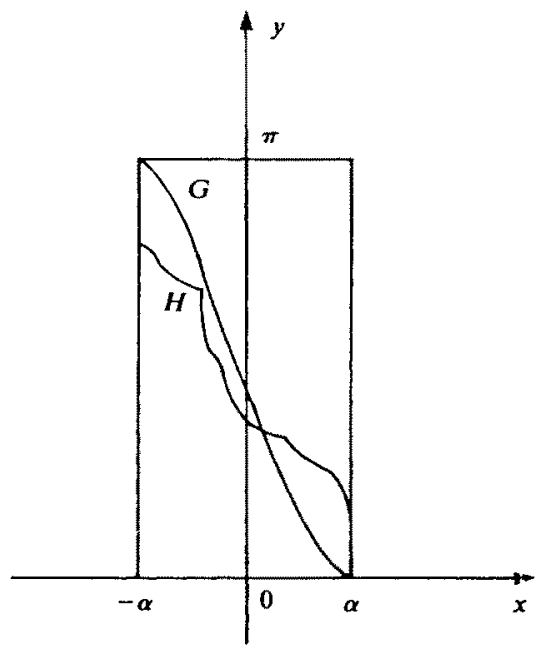

Fig. 3

are tangents $m$ and $n$ to $C_{1}$ at points $M$ and $N$ such that $m \| n,|\angle \vec{x} \overline{M N}| \leq \alpha$, $\mu=\angle \vec{x} \overrightarrow{M N}$, and $\nu=\angle \overrightarrow{M N} m$, where $\angle \overrightarrow{M N} m$ is the angle between $\overrightarrow{M N}$ and $m$ measured by rotating $\overrightarrow{M N}$ counterclockwise into $m$. For any $\mu \in[-\alpha, \alpha]$ there is at least one $\nu \in[0, \pi]$ satisfying $(\mu, \nu) \in H$. The graph of $H$ is a continuous curve connecting the segments $[-\alpha] \times[0, \pi]$ and $[\alpha] \times[0, \pi]$. So $H \cap G \neq 0$. Any pair of lines $m, n$ and points $M, N$ corresponding to an element of $H \cap G$ is a proper choice for the lines $e$ and $f$ and the points $E$ and $F$, respectively.

\section{References}

1. V. A. Zalgaller, The imbedding of one figure in another, Ukrainskij Geometriceskij Sbornik, 10/71 (1971), 19-20 (in Russian).

2. J. Pach, Communication at the problem session, Tagung über konvexe Körper, Oberwolfach, 1984.

3. M. D. Kowaljov, Covering a convex figure by its images under dilatation, Ukrainskij Geometriceskij Sbornik, 27/84 (1984), 57-68 (in Russian).

4. H. E. Debrunner and P. Mani-Levitska, Can you cover your shadows?, Discrete \& Computational Geometry, 1 (1986), 45-58.

Received December 12, 1987. 